\title{
Pulmonary artery hypertension: pertinent vasomotorial cytokines
}

\author{
Shi-Min Yuan \\ Department of Cardiothoracic Surgery, The First Hospital of Putian, Teaching Hospital, Fujian Medical University, Putian 351100, Fujian Province, \\ People's Republic of China
}

Correspondence: Shi-Min Yuan. Department of Cardiothoracic Surgery, The First Hospital of Putian, Teaching Hospital, Fujian Medical University, Putian 351100, Fujian Province, People's Republic of China

$<$ shiminyuan@126.com>

Accepted for publication December 15, 2016

To cite this article: Yuan SM. Pulmonary artery hypertension: pertinent vasomotorial cytokines. Eur. Cytokine Netw. 2017; 28(1): 1-7 doi:10.1684/ecn.2016.0386

\begin{abstract}
Pulmonary artery hypertension is a syndrome that shows similar clinical and pathophysiological features characterized by elevated pulmonary arterial pressure and resistance. There have been a series of hypotheses trying to describe the development of pulmonary artery hypertension; however, none of them perfectly explains its pathogenesis. To highlight the pathogenesis, novel vasomotorial cytokines including hypoxia-inducible factor$1 \alpha$, endothelin-1, urotensin II, Krüppel-like factor 4, calcitonin gene-related peptide, angiopoietins and serotonin closely related to pulmonary artery hypertension are discussed. The development of the new agents relating to these cytokines may improve the relevant treatment strategies.
\end{abstract}

Key words: cytokines, pathogenesis, pulmonary hypertension

Pulmonary arterial hypertension (PAH) is defined as an elevation in mean pulmonary arterial pressure above 25 $\mathrm{mmHg}$ at rest or $30 \mathrm{mmHg}$ with exercise [1]. PAH develops as a result of the pulmonary arterial lumen occlusion due to promoted smooth muscle cell (SMC) proliferation and migration [2]. A series of hypotheses have been proposed to explain this vascular disorder; however, none of them has perfectly interpreted its pathogenesis.

It has been noted that right heart failure is a common cause of death of patients with severe $\mathrm{PAH}$, and right ventricular dysfunction has also been associated with increased mortality in patients with left ventricular failure [3]. Pathogenic pathways, involving right ventricular hypertrophy and evolving toward right heart failure, have been found in myocardium in relation to substrate use, oxidative phosphorylation and high-energy transfer mechanisms [4]. Moreover, in the failing right ventricle, the genes encoding the proteins of the ubiquitin proteasome pathways are highly overexpressed. Treatment with carvedilol, one of the $\beta$-blockers, had cardioprotective and preventive effects on monocrotaline-induced lung remodeling and brought about an increase of right ventricular fetal gene reactivation, an increase of protein kinase $\mathrm{G}$ activity and a reduction of capillary rarefaction and fibrosis, leading to normal expressions of the genes and the proteins. Metoprolol had similar, but less pronounced cardioprotective other than lung remodeling protective effect. These results reflected a highly activated ubiquitin proteasome system in the right ventricular failure patients $[5,6]$.

Nowadays, attention has been focused on how the vasomotorial cytokines function in the underlying signaling pathways that govern the cellular activities (figure 1). Imbalance between the vasodilating and vasoconstrictive cytokines is regarded as an initiating step of development of PAH. Nonetheless, the causal and/or protective roles that the vasomotorial cytokines play in the development of PAH have not been fully elucidated. This article is to discuss some novel vasomotorial cytokines relating to the pathogenesis of PAH.

\section{HYPOXIA-INDUCIBLE FACTOR (HIF)-1}

The pathogenesis of hypoxic PAH has been investigated toward the pivotal role of HIF- $1 \alpha$ in the pulmonary vascular remodeling and the progression of $\mathrm{PAH}$, which are considered to be probably through the regulation of endothelin (ET)-1 and adrenomedullin (ADM) expressions at the transcription level [7]. HIF- $1 \alpha$ involves the transcriptional regulation of $\mathrm{ADM}$ by binding to the correspondent promoter. This has been evidenced in the experiments in adult rats where ADM mRNA was upregulated under hypoxic conditions, with the expressions being the most notable in the arterial endothelial and vascular SMCs. Enhanced ADM expressions abated vascular tension as well as the hypoxia-induced damage to the lung tissues. The early hypoxic induction of upregulation of HIF- $1 \alpha$ and ADM mRNA expressions probably explains the casually direct relationships between HIF- $1 \alpha$ and ADM mRNA expressions, by which synthesis and secretion of ADM in the endothelium and SMCs are increased. Cellular adaptation to prolonged hypoxia may cause vascular remodeling 


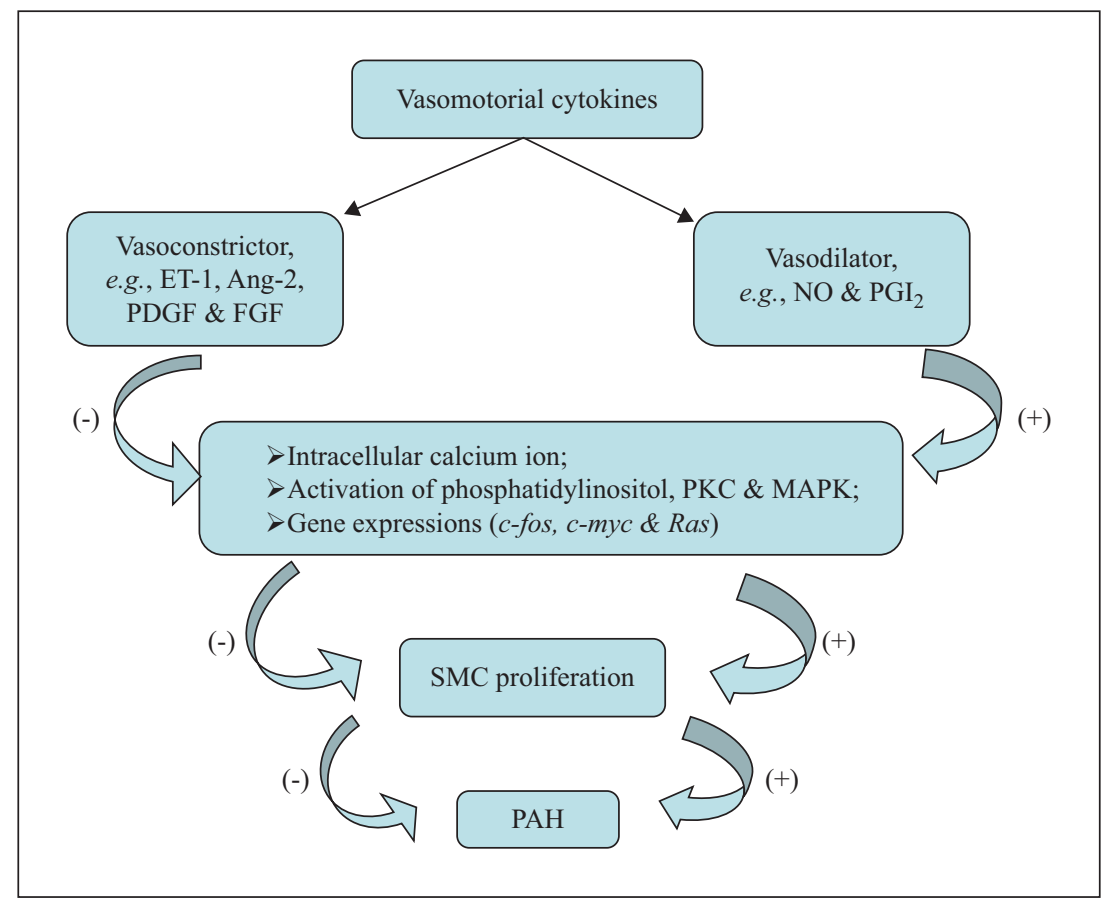

Figure 1

Functions of vasomotorial cytokines: +: enhance; -: attenuate; ET: endothelin; Ang: angiopoietin; PDGF: platelet-derived growth factor; FGF: fibroblast growth factors; NO: nitric oxide; PGI $_{2}$ : prostacyclin; PKC: protein kinase C; MAPK: mitogen activated protein kinase; SMC: smooth muscle cell; PAH: pulmonary artery hypertension.

and endothelial damage leading to a gradual decrease of ADM synthesis and inhibition of ADM on vascular SMC proliferation [7]. ADM is a newly discovered vasoactive peptide, composed of 52 amino acids. ADM is a kind of endothelium-derived relaxing factor, and ADM mRNA is expressed in vascular SMC [8]. The 16th and 21st cysteines form a disulfide bond further forming a circular structure with 6 amino acids. Along with amidation of C-terminal tyrosine residues, these structures show similarity to those of calcitonin gene-related peptide (CGRP) and amylin. Besides, the amino acid sequences of ADM and CGRP share mild homology. Therefore, they all are considered to belong to the CGRP family, and the important sites for ADM to bind receptors and to stimulate the production of cyclic adenosine monophosphate. It has been discovered that multiple mechanisms are involved in the regulation of ADM. The cyclic adenosine monophosphate levels regulate the synthesis of ADM; thrombin, vasoactive intestinal peptide, and interferon- $\gamma$ inhibit ADM synthesis; angiopoietin (Ang)-2, ET-1, bradykinin, and substance P stimulate vascular SMC to produce ADM; glucocorticoid and thyroid hormone upregulate the expression of ADM mRNA in the vascular endothelial cells and vascular SMC [8]. Vascular wall pressure can also induce the upregulation of ADM mRNA in a time- and intensity-dependent fashion. In the hypoxic PAH rats, ADM and its receptors in the lung tissue were observed to be upregulated and their serum levels were also increased. Continuous administration of ADM to the hypoxic rats could alleviate the pulmonary vascular remodeling and the development of PAH (figure 2) [9].

It suggests that ADM might become a new agent for the treatment of PAH. Besides, Ang-2, prostacyclin, vasoactive intestinal peptide, vascular endothelial growth factor, epidermal growth factor, fibroblast growth factor, transforming growth factor, platelet-activating factor and urotensin II (UTII) are also involved in the development of $\mathrm{PAH}$. Endothelial damage inevitably results in imbalance between vasoconstrictor and vasodilator, for instance, the significant decrease of nitric oxide and prostacyclin, and the increase of ET-1 through different signaling pathways, leading to imbalance between proliferation and apoptosis of pulmonary arterial SMC. The abnormal proliferation and diminished apoptosis of pulmonary arterial SMC cause pulmonary vascular stenosis and occlusion, and eventually PAH [8].

Under normal oxygen conditions, the syntheses and expressions of HIF-1 $\alpha$ last in the cytoplasm; whereas rapid degradations occur when affected by the ubiquitinmediated degradation pathway that regulates both normal and abnormal cellular processes. Nevertheless, under hypoxia, the expressions of HIF-1 $\alpha$ grow at an exponential rate and nucleolus accumulations of HIF- $1 \alpha$ become prominent as a result of inhibition of hydroxylation and proteolytic degradation. The expressions of HIF- $1 \alpha$ mRNA could be highly upregulated during the early hypoxic period; however, with the hypoxic progression till seven days thereafter, the expressions of HIF- $1 \alpha$ mRNA were significantly decreased to a level similar to those of the control. In neonatal rats subjected to a lowconcentration oxygen, remarkably elevated expressions of HIF- $1 \alpha$ mRNA were seen in the lung tissues, but dramatic weakening expressions followed in a later stage of the PAH development [7]. In hypoxia, HIF- $1 \alpha$ and HIF- $2 \alpha$ are induced in adventitial fibroblasts. HIF- $1 \alpha$ and HIF- $2 \alpha$ then interact with common and specific targets that mediate the specific effects on proliferation and migration of adventitial fibroblasts [10]. Systemic loss of a single HIF$1 \alpha$ allele has been shown to attenuate hypoxic PAH [11]. HIF- $1 \alpha$ is a master regulator of transcription in hypoxic 


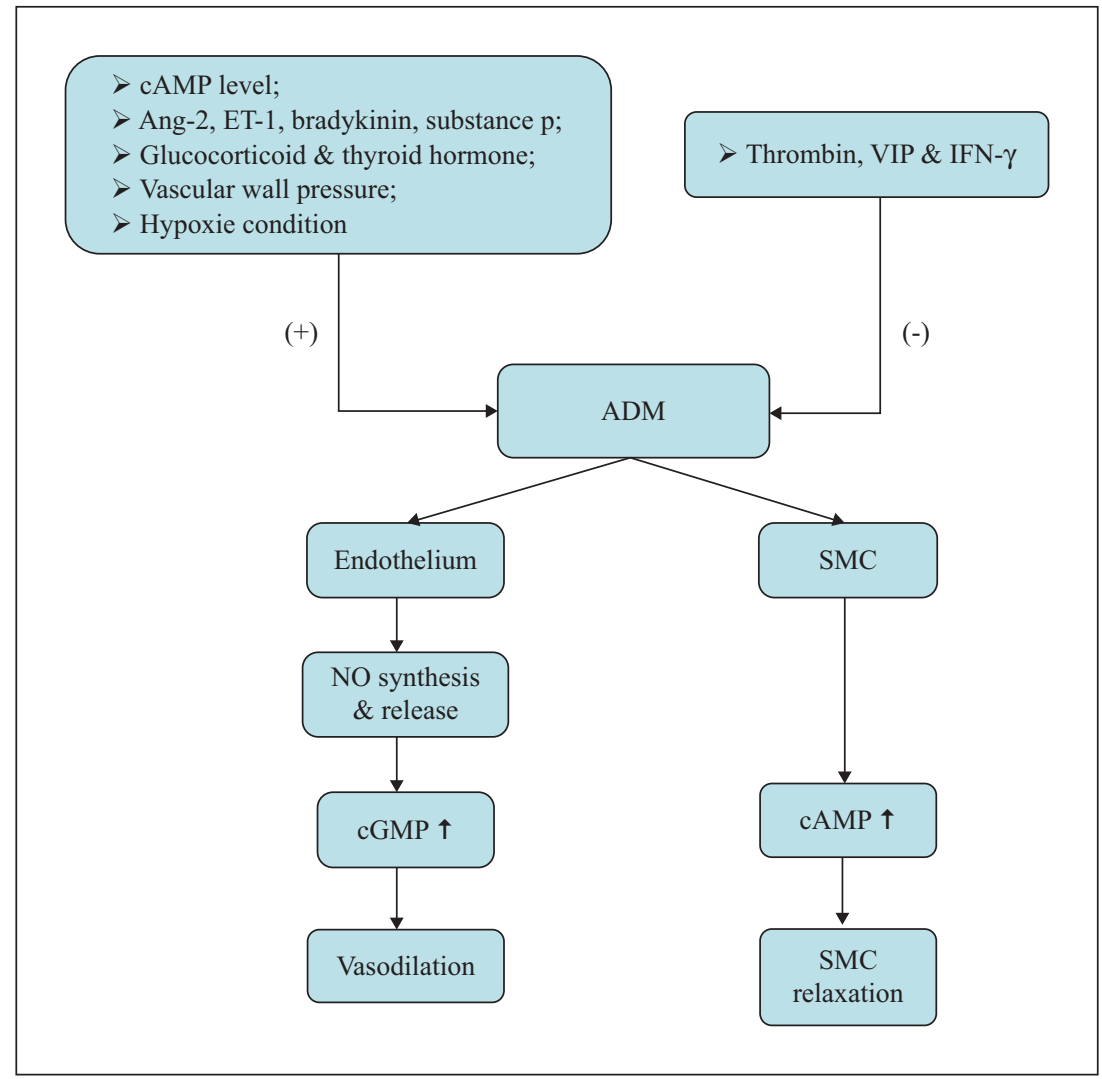

Figure 2

Regulations of adrenomedullin: $\uparrow:$ production increased; +: enhance; -: attenuate; ADM: adrenomedullin; Ang: angiopoietin; cAMP: cyclic adenosine monophosphate; cGMP: cyclic guanosine monophosphate; ET: endothelin; IFN: interferon; NO: nitric oxide; SMC: smooth muscle cell; VIP: vasoactive intestinal peptide.

cells, upregulating genes involved in energy metabolism, proliferation and extracellular matrix reorganization [11]. HIF- $1 \alpha$ plays a role in maintaining the normally low pulmonary vascular tone by decreasing myosin light chain phosphorylation (pMLC) in SMCs to alleviate the occurrence of hypoxic PAH. Loss of HIF- $1 \alpha$ in pulmonary artery SMCs increased pulmonary vascular tone and pMLC in both normoxia and hypoxia [12]. Similarly, observations also showed that selective deletion of HIF-1 $\alpha$ in SMCs increased pulmonary vascular tone under both normoxic and hypoxic conditions as well. Therefore, downregulation of HIF- $1 \alpha$ expression in the pulmonary vasculature may precipitate the development of PAH. Consecutively, cellspecific modulation of HIF- $1 \alpha$ could represent a potential therapeutic strategy for $\mathrm{PAH}$.

\section{ET-1}

ET is a potent vasoconstrictor that constricts blood vessels and elevates blood pressure [13]. The pleiotropic effects of ET-1 involved in the autocrine and paracrine signaling pathways are responsible for the basal vascular tone [14]. ET-1 is increasingly secreted by the damaged endothelial cells. As is mentioned above, at an early hypoxic stage, it promotes the secretion of HIF- $1 \alpha$, further inducing the significant expressions of the HIF-1 $\alpha$ target genes including ET-1. The severe endothelial damage even at the late hypoxic stage also contributes to a sustained low-level secretion of ET-1, followed by a less severe degradation of ET-1 in the lung tissues. It is also conversely the same as ET-1 increases HIF-1 $\alpha$ expression in pulmonary artery SMCs, which is dose dependent. ET-1 regulates calcineurin, a calcium- and calmodulindependent serine/threonine protein phosphatase, also in a dose-dependent manner. ET receptor A, one of the vasoconstrictor subtypes, has specific effects for mediating ET-1 [15]. Enhanced ET activity is closely related to PAH severity, and the potential ET receptor antagonists are already in clinical use for the treatment of PAH [16]. In $\mathrm{PAH}$, the body produces excess ET, contributing to the constriction of blood vessels and affecting the blood pressure in the lungs. Although ET is present in healthy people, high concentrations of the substance have been found in the plasma and lungs of patients with PAH suggesting it is capable of causing PAH or increasing the symptoms of PAH. Two separate receptors of ET have been identified: type A receptors, which induce vasoconstriction by increasing intracellular calcium, most commonly found on vascular SMCs; and type B receptors, located on endothelial cells, stimulate the release of vasodilating agents, such as nitric oxide and prostacyclin. Theoretically, either selective blocking of ET type A receptors alone or nonselective blocking of both ET type A and ET type B receptors together has vasodilating functions as a result of relaxation of vascular SMCs [17]. Evidence suggested that ET was overexpressed in the lungs of patients with PAH and elevated plasma ET concentrations were associated with poor prognosis [18].

ET receptor antagonists are a new class of drugs for the treatment of many diseases, including PAH. ET receptor antagonists are potent vasodilators and antimitotic 
substances, which could specifically dilate and remodel the pulmonary artery, and have recently been proposed as an alternative therapy [17]. ET receptor antagonists have become the mainstays of the treatment of PAH. Three substances are currently available for the treatment of PAH. One of these substances, bosentan, blocks both ET type $\mathrm{A}$ and type B receptors; whereas two other compounds, sitaxsentan and ambrisentan, are more selective blockers of the ET receptor [19].

\section{UII}

Human UII is a cyclic undecapeptide, mediated by a specific cell surface G-protein-coupled receptor, the UII receptor, called UT [20, 21]. UII is considered to be the strongest endogenous vasoconstrictor with similar physiological mechanisms to ET-1. UII also impacts broadly on alternative systems in terms of proliferations of SMCs, fibroblasts, and cancer cells. There has been increasing evidence for UII's role in regulating respiratory physiology [22, 23]. Similarly, UII receptor expression has been localized to rat airway SMCs where it functions as a growth factor [24, 25]. Furthermore, UII can mediate smooth muscle contraction in isolated respiratory tract and pulmonary arteries in primates [26-28]. Elevated plasma levels of UII as well as increased UII and urotensin expressions have been observed in a number of diseased conditions, including PAH as well as other cardiovascular and systemic disorders [29]. UII is highly expressed in the endothelial cells and lymphocytes, stimulating vascular cell adhesion molecule-1 and intercellular adhesion molecule-1 expressions in the endothelium. UII produces reactive oxygen species through nicotinamide adenine dinucleotide phosphate (NADPH) oxidase activation in human vascular SMCs, and thus stimulates SMC proliferations especially showing a synergistic effect in the combination of reactive oxygen species, oxidized low-density lipoprotein and serotonin [30]. UII, by binding to the G-protein-coupled UII receptor, regulates the vascular tone and the signaling cascades of reactive oxygen species and nitric oxide, activates mitogen-activated protein (MAP) kinases, modulates gene expression, stimulates proliferative processes and affects the extracellular matrix [31]. Human UII is a novel activator of NADPH oxidase in human pulmonary artery SMCs, upregulating the expressions of the NADPH oxidase subunits $\mathrm{p} 22$ phox and NOX4 and reactive oxygen species. These substances can be annihilated by transfecting p22phox or NOX4 antisense vectors, which may contribute to a series of complex UII-induced signaling pathways involving extracellular signal-regulated kinase 1/2, p38 MAP kinase, c-Jun N-terminal kinase and Akt, increase the expression of plasminogen activator inhibitor1 and enhance pulmonary artery SMC proliferation in an NADPH oxidase- and kinase-dependent manner [32]. Rong et al. [33] investigated the UII protein expression in patients with congenital heart disease with PAH and found that UII in the lung tissue of the mild PAH group patients was higher than that of patients with a normal pulmonary pressure, but less than that of the severe or moderate PAH group patients.

In a rat model of PAH, palosuran, an antagonist of UII, significantly reduced the UII as well as the ET1 and transforming growth factor- $\beta_{1}$ levels, indicating that palosuran might be an optional treatment alternative for PAH [34]. GSK1440115 is a potent, competitive and selective inhibitor of UII, which mediates UIIinduced systemic pressor response in anesthetized cats [35].

\section{KRÜPPEL-LIKE FACTOR 4 (KLF4)}

KLF4 is a transcriptional factor expressed in the vascular endothelium. It promotes anti-inflammatory and anticoagulant states, and increases nitric oxide synthase expression in the endothelium. KLF4 is expressed in the arterial and venous endothelial cells of the pulmonary circulation, and that KLF4 upregulates the expression and increased the activity of endothelial nitric oxide synthase [36]. KLF4 knockdown resulted in increased pulmonary expression of ET-1 and decreased expression of endothelial nitric oxide synthase, ET type B receptor and prostacyclin synthase. Significant reductions of KLF4 mRNA and protein were noted in the lung tissues of the patients with idiopathic PAH in comparison with the normal control [37, 38]. After hypoxia, right ventricular and pulmonary artery pressures were significantly higher in the KLF4 knockdown animals than in the controls. Knockdown animals also had more severe pulmonary vascular muscularization and right ventricular hypertrophy. KLF4 knockdown resulted in increased pulmonary expression of ET-1 and decreased expression of endothelial nitric oxide synthase, ET type B receptor and prostacyclin synthase [37]. Endothelial KLF4 was proved to be a regulator of neointimal hyperplasia induced by vascular damage through anti-inflammatory and antiproliferative profiles, possibly due to the inhibition of nuclear factor- $\mathrm{B}$ and by affecting some of the coactivators including vascular cell adhesion molecule-1 [39].

\section{CGRP}

Similar to nitric oxide, CGRP is a potent vasodilator, inhibiting hypoxia-induced proliferation of pulmonary artery SMCs dose-dependently, decreasing significantly the expressions of phosphorylated ERK1/2 and collagens I and III, thereby inhibiting phosphorylation of ERK1/2 and alleviating the collagen accumulation of pulmonary arteries [40]. CGRP is believed to play an important role in maintaining low pulmonary vascular resistance and may be involved in modulating the pulmonary vascular response to chronic hypoxia [41]. The increase in pulmonary arterial pressure, pulmonary vascular resistance, right ventricular mass and pulmonary vascular remodeling in response to chronic hypoxia was attenuated in animals overexpressing CGRP [41]. CGRP has a potent vasodilatory effect in the pulmonary arteries and veins contributable to its receptors in the endothelium of the pulmonary artery. CGPR leads to a concentrationdependent relaxation of the pulmonary artery and dilation of precontracted pulmonary artery [42]. Sensory CGRP depletion in PAH rats resulted in decreased p27 and increased phosphorylated ERK1/2, c-fos and $c$-myc expressions; on the contrary, exogenous CGRP application was associated with increased $p 27$ expression and decreased expression of phosphorylated ERK1/2, $c$-fos, and $c$-myc, 
indicating that CGRP inhibits hypoxia-induced proliferation of pulmonary artery SMCs via the ERK1/2/p27/cfos/c-myc pathway [43]. In the hypoxic rat models, blood CGRP was reduced, and reexposure to hypoxia was accompanied by a further decrease of blood CGRP [44]. Bartosik et al. [45] found that the patients with elevated pulmonary artery pressure showed higher plasma CGRP than those with normal pulmonary artery pressure. There was a positive relation between plasma CGRP and pulmonary artery pressure or erythrocyte sedimentation rate. These results provided with solid evidence of CGRP in the pathogenesis of PAH.

\section{ANGS}

Angs are a family member of growth factor, playing special roles in angiogenesis and vascular structural maturation. Ang-1 is one of the Angs, encoded by the ANGPT1 gene, with important properties in vascular development and angiogenesis. During the vascular remodeling process in PAH subjects, Ang-1 was significantly decreased in the pulmonary arteries along with a reduction of Tie-2, a novel endothelium-specific receptor tyrosine kinase, essential for the embryonic vasculature development, and of vascular endothelial growth factor while Tie-2 receptor was inactivated in the lung tissues [46]. It was hypothesized that tonic activation of Tie-2 might play an important role in protecting the pulmonary vasculature by preventing endothelial cell activation in response to toxic stimuli. Moreover, alterations in expression of components of the Ang system, specifically an increase in Ang-2 or a decrease in Tie-2, might contribute to the development of PAH. The downregulated pulmonary Tie-2 expression and variable changes in Ang-1 and Ang-2 have been found increased after exposure to hypoxia [47]. Increased Ang-1 in PAH subjects reflected an insufficient compensatory response [48]. Clinical studies demonstrated a negative correlation between serum Ang-1 levels and the degree of PAH [49]. Ang-1 and associated activation of its Tie- 2 receptor could not be detected in normal human lung samples, but were highly elevated in lung samples from the patients with PAH [48]. Histological investigations illustrated upregulated expressions of Ang-2 mRNA and protein in the plexiform lesions from the lung tissues of idiopathic PAH patients. Hence, Ang-2 might also be involved in the pathogenesis of idiopathic $\mathrm{PAH}$, and plasma Ang-2 might serve as a promising novel biomarker for evaluating the disease severity and the response to treatment in the patients with idiopathic $\mathrm{PAH}$ [50]. The expression of Ang-3 was significantly increased in the vascular intima in the condition of hypoxia. Downregulations of both Ang-1/Tie-2 and vascular endothelial growth factor and upregulation of Ang-3 appeared to be involved in the rarefying vasculature and hypoxic PAH evolution [46]. It was reported that Tie-2 receptor was fourfold higher in lungs and pulmonary endothelial cells from the patients with idiopathic PAH than in those from the control. No significant differences were noted in the Ang-1 and Ang-2 expressions between the lung tissues, pulmonary endothelial cells, and pulmonary artery SMCs. Therefore, the Ang-1/Tie-2 pathway might contribute to pulmonary artery SMC hyperplasia through the activation of the growth factor synthesis in the endothelium of the pulmonary arteries [51].

\section{SEROTONIN AND RECEPTORS}

Serotonin is another vasoconstrictor as well as a mitogen of vascular SMCs, participating in the proliferation and remodeling of pulmonary arterial SMCs during the progression of PAH [52]. Serotonin is synthesized, through tryprophan hydroxylase 1 , in the endothelial cells of the pulmonary artery and can then act on underlying pulmonary arterial SMCs and fibroblasts in a paracrine fashion causing constriction and remodeling [53]. Serotonin exerts bidirectional modulatory effects. First, it can induce the corresponding receptor in the cell signal transduction mechanism, such as calcium ion increase, and activation of phosphatidylinositol, protein kinase $\mathrm{C}$ and mitogen-activated protein kinase (MAPK), etc., and upregulate the expression of c-myc gene, thereby promoting the proliferation of vascular SMC; on the other hand, it can increase the numbers of cyclic adenosine monophosphate receptors on the cellular surface by which it inhibits the proliferation of vascular SMC [8]. Serotonin induces pulmonary vasoconstriction through the interaction with serotonin $1 \mathrm{~B}$ receptors, less so with serotonin $2 \mathrm{~A}$ receptors, which seem to be predominantly involved in systemic vasoconstriction and proliferation [54]. Ang-1 increases the amounts of serotonin available to SMCs and fibroblasts [55]. Raised serum serotonin levels, enhanced expressions of serotonin transporters and serotonin transporter genetic polymorphism were susceptible to clinical PAH [52]. Prolonged exposure to serotonin may lead to PAH by activating constrictor serotonin $1 \mathrm{~B}$ receptors and proliferative serotonin $2 \mathrm{~B}$ receptors [56]. The immunoreactivity for serotonin $1 \mathrm{~B}$ receptors was increased in the media of the pulmonary arteries in 3-week hypoxic rats, especially in those adjacent to the adventitia [57]. Serotonin may promote pulmonary artery SMC proliferation, pulmonary arterial vasoconstriction and local microthrombosis formation [58]. Alterations in platelet serotonin reserve and/or increased platelet depletion may, however, initiate the development of primary PAH [59]. Serotonin may enter pulmonary artery SMCs through the interaction with a specific transporter, resulting in proliferation. Mice lacking the serotonin transporter or treated with serotonin transporter inhibitors are protected against hypoxic PAH [54].

\section{CONCLUSIONS}

PAH is a syndrome with similar clinical and pathophysiological features with complex mechanisms. The cytokines that might participate in the pathogenesis of PAH as mentioned above can be regarded as either a vasoconstrictor or a vasodilator, playing important pathogenic roles through key signaling pathways, particularly the growth factor signaling pathways. The possible mechanisms of the vasomotorial cytokines for vascular remodeling process become more conspicuous by studying the regulations of ADM and ET, the two commonly involved in the signaling pathways relating to $\mathrm{PAH}$ development. The regulations of the cytokines are crucial to the vascular damage and remodeling of the pulmonary arteries. Imbalance between the vasodilating and vasoconstrictive cytokines is regarded as an initiating step of development of PAH. Better understanding of the nature and properties of the cytokines in the development of PAH would be helpful in generating 
new agents that may antagonize or analogize the pathways responsible for $\mathrm{PAH}$, and may enhance the treatment strategies.

Disclosure. Financial support: none. Conflict of interest: none.

\section{REFERENCES}

1. Rabinovitch M. Molecular pathogenesis of pulmonary arterial hypertension. J Clin Invest 2012; 122: 4306-13.

2. Archer SL, Weir EK, Wilkins MR. Basic science of pulmonary arterial hypertension for clinicians: new concepts and experimental therapies. Circulation 2010; 121:2045-66

3. Haddad F, Doyle R, Murphy DJ, Hunt SA. Right ventricular function in cardiovascular disease, part II: pathophysiology, clinicalimportance, and management of right ventricular failure. Circulation 2008; 117: 1717-31.

4. Neubauer S. The failing heart - an engine out of fuel. $N$ Engl J Med 2007; 356: 1140-51.

5. Drake JI, Gomez-Arroyo J, Dumur CI, et al. Chronic carvedilol treatment partially reverses the right ventricular failure transcriptional profile in experimental pulmonary hypertension. Physiol Genomics 2013; 45: 449-61.

6. Bogaard HJ, Natarajan R, Mizuno S, et al. Adrenergic receptor blockade reverses right heart remodeling and dysfunction in pulmonary hypertensive rats. Am J Respir Crit Care Med 2010; 182: 652-60.

7. Wang L, Zhou Y, Li M, Zhu Y. Expression of hypoxiainducible factor- $1 \alpha$, endothelin-1 and adrenomedullin in newborn rats with hypoxia-induced pulmonary hypertension. Exp Ther Med 2014; 8:335-9.

8. Pang YS, Chen MW, Han YL, Zeng M. Pulmonary arterial smooth muscle cell and pulmonary artery hypertension. J Appl Chin Pediatr 2009; $24: 1046-8$

9. Nakanishi K, Osada H, Uenoyama M, et al. Expressions of adrenomedullin mRNA and protein in rats with hypobaric hypoxiainduced pulmonary hypertension. Am J Physiol Heart Circ Physiol 2004; 286: H2159-68.

10. Eul B, Rose F, Krick S, et al. Impact of HIF-1 $\alpha$ and HIF-2 $\alpha$ on proliferation and migration of human pulmonary artery fibroblasts in hypoxia. FASEB J 2006; 20: 163-5.

11. Ball MK, Waypa GB, Mungai PT, et al. Regulation of hypoxiainduced pulmonary hypertension by vascular smooth muscle hypoxia-inducible factor- $1 \alpha$. Am $J$ Respir Crit Care Med 2014; 189: 314-24.

12. Kim YM, Barnes EA, Alvira CM, Ying L, Reddy S, Cornfield DN. Hypoxia-inducible factor- $1 \alpha$ in pulmonary artery smooth muscle cells lowers vascular tone by decreasing myosin light chain phosphorylation. Circ Res 2013; 112: 1230-3.

13. Pinto-Sietsma SJ, Paul M. A role for endothelin in the pathogenesis of hypertension: fact or fiction? Kidney Int Suppl 1998; 67: S1 15-121.

14. Rosanò L, Spinella F, Bagnato A. Endothelin 1 in cancer: biological implications and therapeutic opportunities. Nat Rev Cancer 2013; 13: 637-51.

15. Li M, Liu Y, Jin F, et al. Endothelin-1 induces hypoxia inducible factor $1 \alpha$ expression in pulmonary artery smooth muscle cells. FEBS Lett 2012; 586: 3888-93. doi: 10.1016/j.febslet.2012.08.036.

[16].Satwiko MG, Ikeda K, Nakayama K, et al. Targeted activation of endothelin-1 exacerbates hypoxia-induced pulmonary hypertension. Biochem Biophys Res Commun 2015;465:356-62. doi: 10.1016/j.bbrc.2015.08.002.
17. Liu C, Chen J, Gao Y, Deng B, Liu K. Endothelin receptor antagonists for pulmonary arterial hypertension. Cochrane Database Syst Rev 2013; 2: CD004434.

18. Rubens C, Ewert R, Halank M, Wensel R, Orzechowski HD, Schultheiss HP. Big endothelin-1 and endothelin-1 plasma levels are correlated with the severity of primary pulmonary hypertension. Chest 2001; 120: 1562-9.

19. Dupuis J, Hoeper MM. Endothelin receptor antagonists in pulmonary arterial hypertension. Eur Respir J 2008; 31: 407-15.

20. Ames RS, Sarau HM, Chambers JK, et al. Human urotensin-II is a potent vasoconstrictor and agonist for the orphan receptor GPR14. Nature 1999; 401: 282-6.

21. [No authors listed]. Guide to Receptors and Channels (GRAC), 4th Edition. Br J Pharmacol 2009; 158: S1-254.

22. Zhang Y, Li J, Cao J, et al. Effect of chronic hypoxia on contents of urotensin II and its functional receptors in rat myocardium. Heart Vessels 2002; 16: 64-8.

23. Qi J, Du J, Tang X, Li J, Wei B, Tang C. The upregulation of endothelial nitric oxide synthase and UTII is associated with PAH and vascular diseases in rats produced by aortocaval shunting. Heart Vessels 2004; 19: 81-8.

24. Chen YH, Zhao MW, Yao WZ, Pang YZ, Tang CS. The signal transduction pathway in the proliferation of airway smooth muscle cells induced by urotensin II. Chin Med J (Engl) 2004; 117 : $37-41$.

25. Zhang WX, Liang YF, Wang XM, et al. Urotensin upregulates transforming growth factor- $\beta_{1}$ expression of asthma airway through ERK-dependent pathway. Mol Cell Biochem 2012;364: 291-8.

26. Douglas SA, Sulpizio AC, Piercy V, et al. Differential vasoconstrictor activity of human urotensin-II in vascular tissue isolated from the rat, mouse, dog, pig, marmoset and cynomolgus monkey. $\mathrm{Br} \mathrm{J}$ Pharmacol 2000; 131: 1262-74.

27. Hay DW, Luttmann MA, Douglas SA. Human urotensin-II is a potent spasmogen of primate airway smooth muscle. Br J Pharmacol 2000; 131: 10-2.

28. MacLean MR, Alexander D, Stirrat A, et al. Contractile responses to human urotensin-II in rat and human pulmonary arteries: effect of endothelial factors and chronic hypoxia in the rat. Br J Pharmacol 2000; 130: 201-4.

29. Ross B, McKendy K, Giaid A. Role of urotensin II in health and disease. Am J Physiol Regul Integr Comp Physiol 2010; 298: R1156-72.

30. Watanabe T, Arita S, Shiraishi Y, et al. Human urotensin II promotes hypertension and atherosclerotic cardiovascular diseases. Curr Med Chem 2009; 16: 550-63.

31. Djordjevic T, Görlach A. Urotensin-II in the lung: a matter for vascular remodelling and pulmonary hypertension? Thromb Haemost 2007; 98: 952-62, http://dx.doi.org/10.1160/TH07-04-0294.

32. Djordjevic T, BelAiba RS, Bonello S, Pfeilschifter J, Hess J, Görlach A. Human urotensin II is a novel activator of NADPH oxidase in human pulmonary artery smooth muscle cells. Arterioscler Thromb Vasc Biol 2005; 25: 519-25.

33. Rong X, Wu HP, Qiu HX, et al. Expression and role of urotensin II on the lung of patients with pulmonary hypertension with congenital heart disease. Zhonghua Er Ke Za Zhi 2012; 50: 689-91.

34. Onat AM, Pehlivan Y, Turkbeyler IH, et al. Urotensin inhibition with palosuran could be a promising alternative in pulmonary arterial hypertension. Inflammation 2013; 36: 405-12. 
35. Behm DJ, Aiyar NV, Olzinski AR, et al. GSK1562590, a slowly dissociating urotensin-II receptor antagonist, exhibits prolonged pharmacodynamic activity ex vivo. Br J Pharmacol 2010; 161: 207-28.

36. Hamik A, Lin Z, Kumar A, et al. Kruppel-like factor 4 regulates endothelial inflammation. J Biol Chem 2007; 282: 13769-79.

37. Shatat MA, Tian H, Zhang R, et al. Endothelial Krüppel-like factor 4 modulates pulmonary arterial hypertension. Am J Respir Cell Mol Biol 2014; 50: 647-53.

38. Shatat MA, Peachey J, Hamik A, et al. Krüppel-like factor 4 modulates pulmonary arterial endothelial cells modulates smooth muscle cell phenotype. Am J Respir Cell Mol Biol 2016; 193: A2222, http://www.atsjournals.org/doi/abs/10.1164/ajrccm-conference. 2016.193.1_MeetingAbstracts.A2222.

39. Tugal D, Jain MK, Simon DI. Endothelial KLF4: crippling vascular injury? J Am Heart Assoc 2014; 3: e000769.

40. Li XW, Du J, Li YJ. The effect of calcitonin gene-related peptide on collagen accumulation in pulmonary arteries of rats with hypoxic pulmonary arterial hypertension. Zhongguo Ying Yong Sheng Li Xue Za Zhi 2013; 29: 182-6192.

41. Bivalacqua TJ, Hyman AL, Kadowitz PJ, Paolocci N, Kass DA, Champion HC. Role of calcitonin gene-related peptide (CGRP) in chronic hypoxia-induced pulmonary hypertension in the mouse. Influence of gene transfer in vivo. Regul Pept 2002; 108: 129-33.

42. Ghatta S, Nimmagadda D. Calcitonin gene-related peptide: understanding its role. Indian J Pharmacol 2004; 36: 277-83.

43. Li XW, Hu CP, Wu WH, Zhang WF, Zou XZ, Li YJ. Inhibitory effect of calcitonin gene-related peptide on hypoxia-induced rat pulmonary artery smooth muscle cells proliferation: role of ERK1/2 and p27. Eur J Pharmacol 2012; 679: 117-26.

44. Keith IM, Tjen-A-Looi S, Kraiczi H, Ekman R. Three-week neonatal hypoxia reduces blood CGRP and causes persistent pulmonary hypertension in rats. Am J Physiol Heart Circ Physiol 2000; 279: H1571-8.

45. Bartosik I, Eskilsson J, Ekman R, Akesson A, Scheja A. Correlation between plasma concentrations of calcitonin gene related peptide and pulmonary pressure in patients with systemic sclerosis. Ann Rheum Dis 2002; 61:261-3.

46. Yamamoto A, Takahashi H, Kojima Y, et al. Downregulation of angiopoietin-1 and Tie 2 in chronic hypoxic pulmonary hypertension. Respiration 2008; 75: 328-38.
47. Zhao YD, Campbell AI, Robb M, Ng D, Stewart DJ. Protective role of angiopoietin-1 in experimental pulmonary hypertension. Circ Res 2003; 92: 984-91.

48. Rudge JS, Thurston G, Yancopoulos GD. Angiopoietin-1 and pulmonary hypertension: cause or cure? Circ Res 2003;92: 947-9.

49. Karapınar H, Esen O, Emiroğlu Y, et al. Serum levels of angiopoietin-1 in patients with pulmonary hypertension due to mitral stenosis. Heart Vessels 2011; 26: 536-41.

50. Kümpers P, Nickel N, Lukasz A, et al. Circulating angiopoietins in idiopathic pulmonary arterial hypertension. Eur Heart $J$ 2010; 31: 2291-300.

51. Dewachter L, Adnot S, Fadel E, et al. Angiopoietin/Tie2 pathway influences smooth muscle hyperplasia in idiopathic pulmonary hypertension. Am J Respir Crit Care Med 2006; 174: 1025-33.

52. Li BB, Jiang Z. Role of serotonin in proliferation of pulmonary arterial smooth muscle cells and remodeling of pulmonary vasculature. Int J Anesth Resus 2007; 28: 441-4.

53. Dempsie Y, MacLean MR. Pulmonary hypertension: therapeutic targets within the serotonin system. Br J Pharmacol 2008; 155: 455-62.

54. Morecroft I, Loughlin L, Nilsen M, et al. Functional interactions between 5-hydroxytryptamine receptors and the serotonin transporter in pulmonary arteries. $J$ Pharmacol Exp Ther 2005; 313: 539-48.

55. Sullivan CC, Du L, Chu D, et al. Induction of pulmonary hypertension by an angiopoietin 1/TIE2/serotonin pathway. Proc Natl Acad Sci U S A 2003; 100: 12331-6.

56. Kaumann AJ, Levy FO. 5-hydroxytryptamine receptors in the human cardiovascular system. Pharmacol Ther 2006; 111: 674-706.

57. Wang JX, Tang FK, Xiao J, et al. Expression and distribution of 5-HTIB receptors in lung tissue in rats with hypoxic pulmonary hypertension. Chin J Pathophysiol 2010;26: 1579-83.

58. Fanburg BL, Lee SL. A new role for an old molecule: serotonin as a mitogen. Am J Physiol 1997; 272: L795-806.

59. Eddahibi S, Fabre V, Boni C, et al. Induction of serotonin transporter by hypoxia in pulmonary vascular smooth muscle cells. Relationship with the mitogenic action of serotonin. Circ Res 1999; 84: 329-36. 